\title{
Retinal nerve fiber layer thickness in chronic obstructive pulmonary disease: An optical coherence tomography study
}

\author{
Yeap Thye Ghee ${ }^{1}$, Mushawiahti Mustapha', Roslan Harun ${ }^{2}$, Syed \\ Zulkifli Syed Zakaria, Jemaima Che Hamzah², Hairul Nizam Harun², \\ Faridah Hanom Annuar ${ }^{2}$ \\ 'Department of Ophthalmology, Universiti Kebangsaan Malaysia Medical Centre, \\ Kuala Lumpur, Malaysia; ${ }^{2}$ Department of Medicine, Universiti Kebangsaan \\ Malaysia Medical Centre, Kuala Lumpur, Malaysia; ${ }^{3}$ Department of Paediatric, \\ Universiti Kebangsaan Malaysia Medical Centre, Kuala Lumpur, Malaysia
}

\begin{abstract}
Background: To evaluate changes in the retinal nerve fiber layer (RNFL) thickness in patients with chronic obstructive pulmonary disease (COPD) using optical coherence tomography (OCT).

Methods: In this case-controlled study, COPD subjects with various disease durations and severity were recruited. The standard global initiative for chronic obstructive lung disease (GOLD) criteria was used to determine the severity of COPD in the subjects. Healthy individuals were recruited as the control group. Peripapillary RNFL thickness was measured using spectral domain OCT.

Results: Seventy-one eyes of 71 COPD patients and 71 eyes of healthy controls were examined. The RNFL in all quadrants and the average RNFL were significantly thinner in patients with COPD compared to the control group $(p<0.05)$. There were significant correlations between severity of COPD and RNFL thickness $(p<0.05)$ for the average RNFL $(r=0.831)$ and RNFL in all quadrants (superior RNFL $r=0.736$, inferior RNFL $r=0.681$, nasal RNFL $r=0.600$, and temporal RNFL $r=0.353$ ). Similar significant correlations were found between RNFL thickness and duration of COPD $(p<0.005)$ for the average RNFL $(r=$ -0.674) and RNFL in all quadrants (superior $r=-0.615$, inferior $r=-0.472$, nasal $r=-0.484$, and temporal $r=-0.323$ ). The risk of having a thin RNFL (odds ratio) was 13-fold higher in patients with COPD.

Conclusions: These results suggest that COPD causes thinning of the RNFL, and severe retinal nerve fiber injury occurs more frequently in advanced COPD.
\end{abstract}

Keywords: glaucoma; chronic obstructive pulmonary disease; optical coherence tomography; retinal ganglion cells; optic neuropathy

\section{Introduction}

Chronic obstructive pulmonary disease (COPD) is a global health concern with a significant economic and social burden. ${ }^{1}$ The prevalence of moderate to severe COPD in Malaysia in 2003 was estimated by the regional COPD working group to be

Correspondence: Mushawiahti Mustaph, Department of Ophthalmology, Universiti Kebangsaan Malaysia Medical Centre, Jalan Yaacob Latif, 56000 Cheras, Kuala Lumpur, Malaysia.

E-mail: drmusha@yahoo.com or drmusha@gmail.com 
$4.7 \%{ }^{2}$ COPD is characterized by progressive and persistent airflow limitation that is not fully reversible. ${ }^{3}$ It is associated with a chronic inflammatory response in the airways and lung parenchyma to noxious particles or gases particularly cigarette smoking. Although COPD primarily affects the lung, it is also associated with other significant systemic consequences such as hypertension, diabetes, coronary artery disease, congestive heart failure and stroke. ${ }^{3}$

The systemic changes in COPD are attributed to oxidative stress resulting in altered circulating levels of inflammatory mediators and acute phase proteins such as endothelin-1 (ET-1). ${ }^{4}$ ET-1 is produced by the oxidative stress and increased levels have been reported in the plasma and urine of COPD patients. ${ }^{5} \mathrm{ET}-1$ is a potent vasoconstrictor causing systemic vascular effects. ${ }^{1}$ Ozer et al. ${ }^{6}$ reported high resistance indices in most orbital arteries in COPD patients, including the ophthalmic and posterior ciliary arteries, probably due to the increased circulating plasma ET-1 enzymes. Such disturbances in the retrobulbar hemodynamic circulation increase the risk of optic nerve head hypoperfusion and eventually lead to retinal ganglion cell loss.?

Optic nerve abnormality in COPD has been indirectly evaluated by many authors. ${ }^{8}$ Ozge et al. ${ }^{8}$ found significant abnormalities in the optic nerves in $82.1 \%$ of COPD patients compared to healthy controls using visual evoked potential (VEP). They observed prolonged latency and reduced amplitude of $\mathrm{P}_{100}$, which represents axonal and demyelinating dysfunction of the peripheral field of the optic nerve. ${ }^{8}$ Similarly, Gupta et al. ${ }^{9}$ reported prolonged latency and decreased amplitude of $\mathrm{P}_{100}$ in both eyes in patients with stable COPD with no clinical evidence of visual impairment or peripheral neuropathy.

To the best of our knowledge, direct and objective evaluation of the retinal nerve fiber layer (RNFL) in COPD patients has not been reported. We aimed to objectively demonstrate the possible damaging effect of COPD on the RNFL using optical coherence tomography (OCT), the human bio-microscope. We also investigated a possible association between the severity of airflow limitation and the degree of injury to the RNFL, which has not yet been reported.

\section{Methods}

This case-controlled study was conducted in Universiti Kebangsaan Malaysia Medical Centre (UKMMC), Kuala Lumpur. Patients with COPD were recruited from the respiratory clinic who attended their usual follow-up. The control group consisted of healthy volunteers from the hospital staff and relatives accompanying the COPD patients. The study was conducted from March 2013 until March 2014.

Inclusion criteria for the COPD study group were: (i) age of 18 years and older; (ii) diagnosed with COPD by respiratory physician with post-bronchodilator forced expiratory volume in one second to forced vital capacity (FEV1/FVC) ratio of less than 0.70; (iii) intraocular pressure (IOP) $\leq 21 \mathrm{mmHg}$; (iv) open angles on gonioscopic examination; and (v) good OCT signal strength. The healthy individuals in the control group had no significant medical history, an IOP $\leq 21 \mathrm{mmHg}$ and normal angles on gonioscopy. Exclusion criteria for both groups included: (i) glaucoma 
diagnosis; (ii) a previous history of ocular trauma; (iii) myopia $>6.00$ diopter; (iv) previous intracranial injury; and (v) presence of obstructive sleep apnea, diabetes or hypertension.

Written informed consents were obtained from all subjects .The severity of COPD was based on post-bronchodilator FEV1 \% predicted values and classified according to the Global Initiative for Chronic Obstructive Lung Disease (GOLD) staging. In patients with FEV1/FVC ratio $<70 \%$, GOLD 1 (mild COPD) the FEV1 is equal or more than $80 \%$ normal, GOLD 2 (moderate COPD) FEV1 50-79\% normal, GOLD 3 (severe COPD) FEV1 30-49\% normal and GOLD 4 (very severe COPD) FEV1 less than $30 \%$ normal. ${ }^{10} \mathrm{~A}$ higher GOLD classification suggests an increased risk of exacerbation, hospitalization and death as airflow becomes more limited. As COPD is a systemic disease, both eyes were expected to be affected equally, and therefore, only the right eye was selected for evaluation and analysis. In cases where the right eye was not available, the left eye was used. The participants' demographic data, past medical and ocular history were recorded. Visual acuity of each eye was measured using Snellen Chart and complete ocular examination was performed. Dilated fundus examination was performed using condensing lens. Peripapillary RNFL ( $3 \mathrm{~mm}$ around the disc) was measured using spectral domain OCT (Heidelberg Engineering, Heidelberg, Germany).

Statistical analysis was performed using IBM SPSS Statistics for Windows, version 22.0. (IBM Corp, Armonk, NY). Comparisons between the RNFL measurements for COPD and healthy individuals were analyzed using paired t-tests. The associations between the RNFL thickness and severity as well as duration of COPD were analyzed using the Spearman's test.

\section{Results}

Demographic and clinical characteristics of the study group

A total of 142 subjects were recruited ( 71 patients with COPD and 71 subjects for the control group). Seventy-six (53.5\%) subjects were Malay, 59 (41.5\%) were Chinese and seven (5\%) were Indian (Table 1). A total of 108 (76.1\%) subjects were male, and 34 (23.9\%) were female. There were no significant differences in the age, gender, ethnicity and baseline intraocular pressure between the control and COPD group (Table 1). The mean duration of disease was $8.01 \pm 4.46$ years, and ranged from one to 20 years.

Classification was based on the severity of COPD and used the GOLD staging. Sixteen (22.4\%) patients were classified as GOLD 1, 27 (38.0\%) patients as GOLD 2, 21 (29.6\%) patients as GOLD 3 and only seven (9.9\%) patients were classified as the GOLD 4 (Table 1). 
Table 1. Demographic and clinical descriptive statistics

\begin{tabular}{|c|c|c|c|}
\hline Characteristics & $\begin{array}{l}\text { COPD } \\
n=71\end{array}$ & $\begin{array}{c}\text { Control } \\
n=71\end{array}$ & p Value \\
\hline \multicolumn{4}{|l|}{ Age (years) } \\
\hline Mean \pm SD & $68.27 \pm 6.62$ & $69.73 \pm 5.14$ & $0.143^{*}$ \\
\hline \multicolumn{4}{|l|}{ Gender } \\
\hline Male, n(\%) & $55(77.5)$ & $53(74.6)$ & $0.844^{ \pm}$ \\
\hline Female , n (\%) & $16(22.5)$ & $18(25.4)$ & \\
\hline \multicolumn{4}{|l|}{ Ethnicity } \\
\hline Malay & $38(53.5)$ & $38(53.5)$ & $0.923^{f}$ \\
\hline Chinese & $30(42.3)$ & $29(40.8)$ & \\
\hline Indian & $3(4.2)$ & $4(5.6)$ & \\
\hline \multicolumn{4}{|l|}{ IOP, mmHG } \\
\hline Mean \pm SD & $15.58 \pm 1.79$ & $15.11 \pm 1.78$ & $0.064^{*}$ \\
\hline $\begin{array}{l}\text { Duration of COPD } \\
\text { (years), }\end{array}$ & $8.01 \pm 4.46$ & & \\
\hline mean $\pm \mathrm{SD}$ (range) & $(1-20)$ & & \\
\hline \multicolumn{4}{|c|}{ GOLD staging of COPD, $n(\%)$} \\
\hline GOLD 1 & $16(22.5)$ & & \\
\hline GOLD 2 & $27(38.0)$ & & \\
\hline GOLD 3 & $21(29.6)$ & & \\
\hline GOLD 4 & $7(9.9)$ & & \\
\hline
\end{tabular}

*t-test, ${ }^{\ddagger}$ Chi-square, ${ }^{\ddagger}$ Mann-Whitney U-test

\section{Peripapillary RNFL was thinner in COPD patients compared to controls}

In general, the RNFL was significantly thinner in patients with COPD compared to the controls ( $p<0.05 ;$ Fig. 1$)$. The RNFL was significantly thicker in the control group compared to the COPD group in the average RNFL and RNFL in all quadrants: average RNFL (110.73 $\pm 8.47 \mathrm{~mm}$ vs $93.27 \pm 17.41 \mathrm{~mm} ; \mathrm{p}<0.05)$; superior RNFL $(108.56 \pm 24.17 \mathrm{~mm}$ vs $133.17 \pm 15.27 \mathrm{~mm} ; \mathrm{p}<0.05)$; inferior RNFL (144.66 \pm 15.81 $\mathrm{mm}$ vs. $121.76 \pm 33.05 \mathrm{~mm} ; \mathrm{p}<0.05)$; nasal RNFL $(80.03 \pm 13.12 \mathrm{~mm}$ vs $68.21 \pm 16.80$ $\mathrm{mm} ; \mathrm{p}<0.05)$; and temporal RNFL $(85.25 \pm 15.95 \mathrm{~mm}$ vs $71.82 \pm 18.47 \mathrm{~mm} ; \mathrm{p}<0.05)$. 


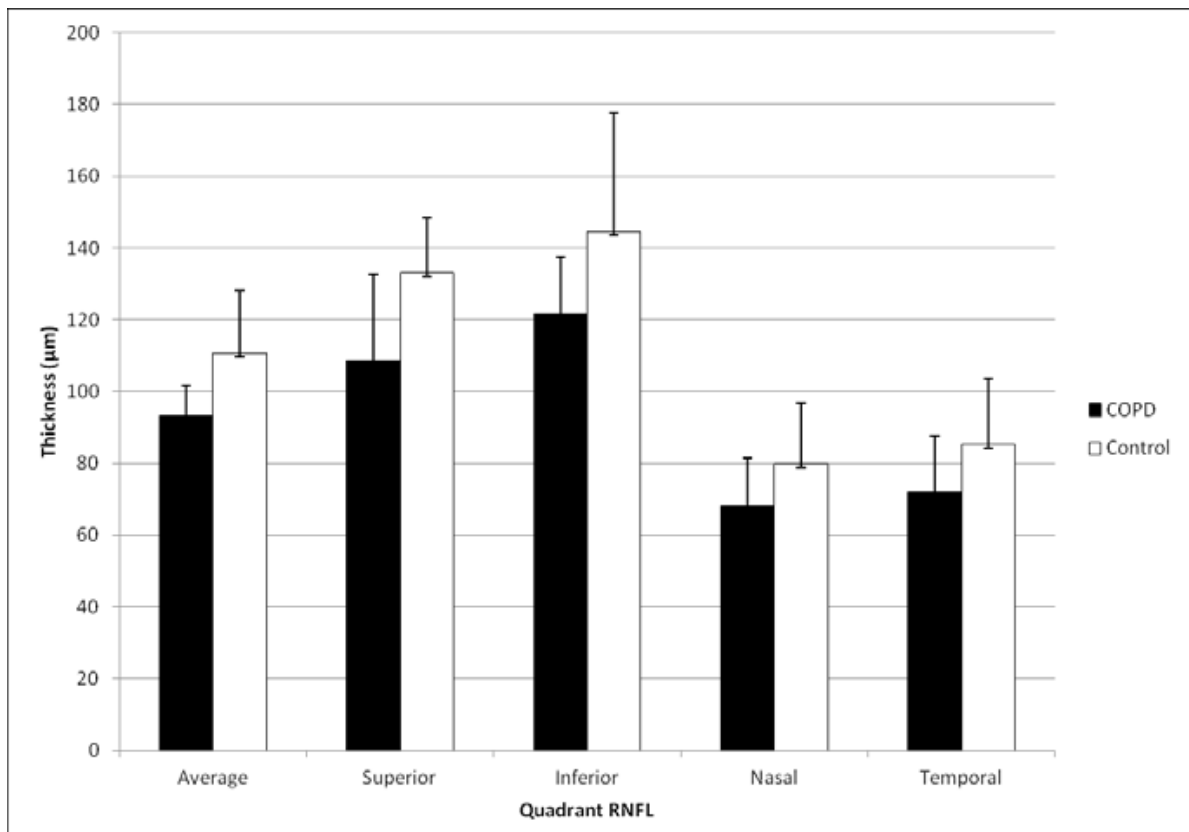

Fig. 1. Peripapillary retinal nerve fiber layer (RNFL) thickness between COPD patients and healthy controls. The average and all quadrant RNFLs are significantly thinner in COPD patients compared to controls.

\section{RNFL thickness and severity of COPD}

The severity of COPD was based on the GOLD staging as determined by the post bronchodilator FEV1. RNFL was significantly thinner in the more severe forms of COPD ( $<<0.05$; Fig. 1). The average RNFL thickness for the control group was $110.73 \pm 8.47 \mathrm{~mm}$. The average RNFL thickness was $105.75 \pm 7.74 \mathrm{~mm}$ for GOLD 1 $(p=0.01), 101.52 \pm 8.73 \mathrm{~mm}$ for GOLD 2, $83.86 \pm 13.91 \mathrm{~mm}$ for GOLD 3, and 61.14 \pm $13.28 \mathrm{~mm}$ for GOLD 4 COPD patients.

Looking at the individual quadrants, the thickness of superior quadrant RNFL in the control group was $133.17 \pm 15.27 \mathrm{~mm}$. Compared to controls, the superior quadrant RNFL thickness in patients with GOLD 1 was $124.94 \pm 13.96 \mathrm{~mm}(p=0.02)$, GOLD 2, $118.30 \pm 13.90 \mathrm{~mm}(p<0.05), G O L D 3,95.29 \pm 23.98 \mathrm{~mm}(p<0.05)$ and GOLD 4, $73.43 \pm 19.40 \mathrm{~mm}(\mathrm{p}<0.05)$. The inferior quadrant RNFL thickness for the control group was $144.66 \pm 15.82 \mathrm{~mm}$. Compared to controls, the inferior RNFL thickness for GOLD 1 was $137.19 \pm 29.08 \mathrm{~mm}(p=0.463)$; GOLD 2, $132.78 \pm 29.28$ $\mathrm{mm}(\mathrm{p}=0.054) ; \mathrm{GOLD} 3,112.10 \pm 26.20 \mathrm{~mm}(\mathrm{p}<0.05)$ and GOLD 4, $73.00 \pm 18.50$ $\mathrm{mm}(\mathrm{p}<0.05)$. The nasal RNFL thickness for the control group was $80.03 \pm 13.12$ $\mathrm{mm}$. The same quadrant in GOLD 1 patients was $77.56 \pm 8.94 \mathrm{~mm}(p=0.408)$; GOLD $2,73.63 \pm 10.54 \mathrm{~mm}(p=0.041) ;$ GOLD 3, $63.86 \pm 14.16(p<0.005)$ and GOLD 4, $39.00 \pm 22.72 \mathrm{~mm}(p<0.05)$. Finally, in the temporal quadrant the RNFL thickness 
in the control group was $85.25 \pm 15.95 \mathrm{~mm}$. The same quadrant in GOLD 1 patients was $77.13 \pm 9.55 \mathrm{~mm}(\mathrm{p}=0.043) ; \mathrm{GOLD} 2,78.22 \pm 10.30 \mathrm{~mm}(\mathrm{p}=0.049) ; \mathrm{GOLD} 3$, $63.76 \pm 20.69(p<0.05)$ and GOLD 4, $59.14 \pm 34.50 \mathrm{~mm}(p=0.033)$.

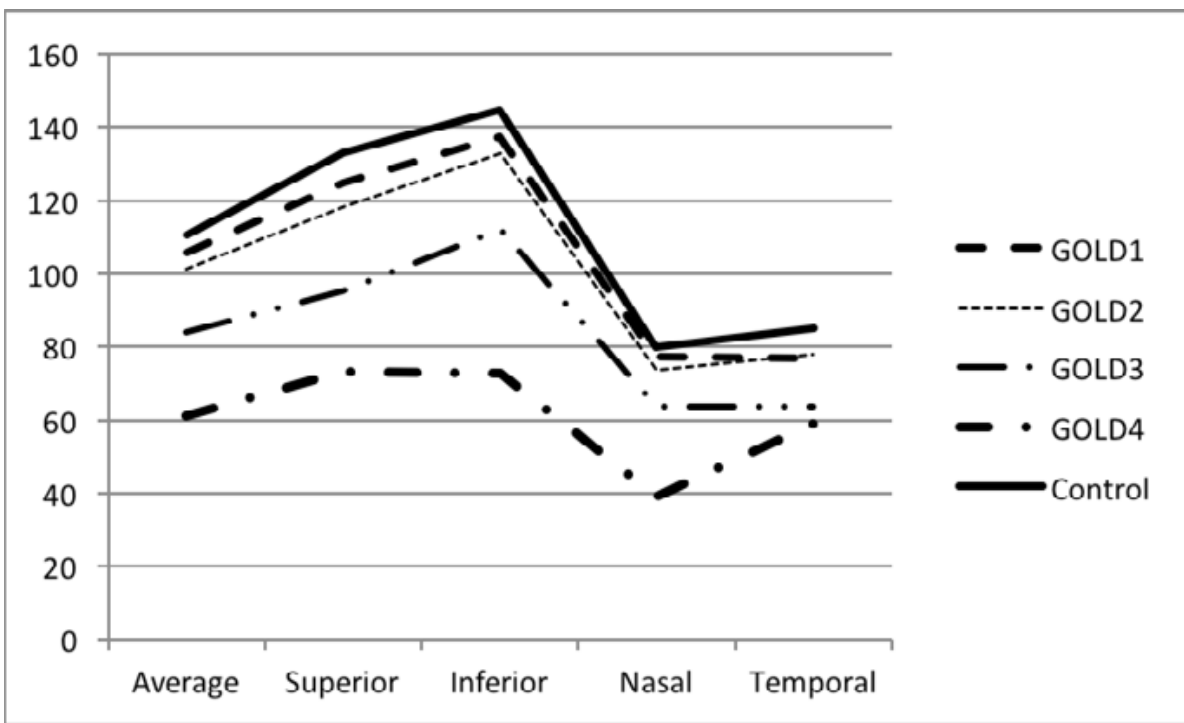

Fig. 2. Retinal nerve fiber layer (RNFL) thickness and COPD severity based on the GOLD staging system (GOLD1 is the least severe form and GOLD4 is the most severe form of COPD). RNFL becomes thinner with increasing severity of COPD.

\section{Correlation between RNFL thickness and duration of COPD}

We also found significant correlations between duration of COPD and RNFL thickness in all quadrants, including average RNFL $(p<0.05)$. Longer duration of COPD was associated with thinner RNFL. Average RNFL thickness demonstrated the strongest correlation $(r=-0.674)$, followed by superior RNFL $(r=-0.615)$, inferior RNFL ( $r=-0.472)$ and nasal RNFL $(r=-0.323)$ thickness. Thickness of the temporal quadrant RNFL showed the lowest level of correlation $(r=-0.323)$ with duration of COPD.

The odds ratio for COPD patients was calculated based on average RNFL. Thin RNFL was defined as $25 \%$ of the average RNFL value (i.e., $94 \mathrm{~mm}$ ). The risk of having a thin retina was 13 -fold higher in patients with COPD (OR 13.7, 95\% Cl: 4.5, 41.8), and this was statistically significant.

\section{Discussion}

COPD is a systemic disorder that is frequently associated with significant extrapulmonary manifestations, which impair the patient's quality of life. ${ }^{3}$ Polyneuropathy has been widely studied asa common systemic disorder in COPD. ${ }^{8,9}$ However, this is the first study to evaluate changes in the retinal nerve fiber layer (RNFL) in 


\section{COPD patients.}

The results of our study demonstrated significant thinning of RNFL in COPD patients compared to healthy individuals. Reduction of RNFL thickness was generally present in all peripapillary quadrants and the average RNFL thickness was also reduced. This strengthens the results reported in previous studies. ${ }^{8}$ Thinning of RNFL indicates diffuse axonal and retinal ganglion cell loss, most likely due to systemic hypoxemia. Peripheral airway obstruction, parenchymal destruction and pulmonary abnormalities in COPD reduce the lung's capacity for gas exchange and result in chronic systemic hypoxemia and hypercapnia. Retinal ganglion cells are highly sensitive to hypoxemia; even a mild decrease in arterial blood oxygen causes retinal ganglion cells to be impaired. ${ }^{11,12}$ Similarly, systemic chronic hypoxemia has previously been shown to cause direct and indirect anoxic damage to the optic nervehead. ${ }^{11}$

While previous studies have shown that hypoxemia in obstructive apnea was associated with normotensive glaucoma (NTG), ${ }^{11}$ the RNFL was found to be diminished in our COPD patients without a diagnosis of glaucoma. There are two current theories to explain the mechanisms leading to open angle glaucoma: a mechanical theory relating to intraocular pressure, and a vascular theory relating to areduction in ocular blood flow. ${ }^{13}$ As ET-1 is a potent vasoconstrictor, the high systemic levels of ET-1 observed in COPD patients may simultaneously reduce ocular blood flow and increase ocular perfusion pressure, leading to progressive damage of the optic nerve. ${ }^{5,6,14}$ Therefore, it is worth considering COPD as an important risk factor for glaucoma and subsequent optic nerve damage.

In this particular study, an experienced respiratory physician performed the clinical COPD diagnosis, and the severity of disease was classified based on the post bronchodilator FEV1 or the standard GOLD spirometric grading systems (GOLD 1-4). GOLD 1 is the least severe form of the disease, while GOLD 4 denotes the most severe form of airflow limitation.We found a strong association between GOLD severity stage and thinning of peripapillary RNFL. Increased resistance in the peripheral airway as the disease advances further compromises the ocular blood flow, which in turn damages the RNFL. While increased duration of the disease also correlated with reduced thickness of the RNFL, the strongest predictor of RNFL thickness was the severity of COPD based on post bronchodilator FEV1 readings.

During the early stages of COPD, a deficiency in relative oxygen levels may provoke a compensatory effect and blood flow velocity will increase to meet the metabolic demand..$^{15}$ This efficient auto-regulation mechanism may protect the retina from early damage. Therefore, the insignificant thinning of inferior and nasal RNFL during the early stages of COPD observed in our study may be due to this auto-regulation mechanism. However, with advancement of disease and time, retinal vascular auto-regulation might fail to compensate for the severe hypoxemic conditions, and eventually the optic nerve head will be damaged.

A limitation of this study was its cross-sectional design, which did not allow us to directly demonstrate causation. Therefore, a larger cohort study in the future would better demonstrate a causal relationship between COPD and thinning of the 
RNFL. Secondly, visual field was not evaluated in our subjects. Clinical screening was used to exclude subjects with possible glaucoma. However, incorporating visual field assessment would have been useful, especially for those with severe COPD who are prone to having a thin RNFL. In the future, it would be interesting to look at pre-perimetric changes before the optic disc changes become evident. Such studies would also provide a more comprehensive assessment of optic nerve head damage in COPD patients.

In conclusion, COPD causes retinal nerve fiber injury and the retinal nerve fiber layer becomes thinner over time. The severity of COPD is a strong predictor for the degree of thinning of the RNFL. As smoking is a major cause of COPD, and we found that COPD increases the risk of optic nerve damage. Indirectly, the results of this study further support the disastrous effect of cigarette smoking to the eye. Our findings are also relevant for ophthalmologists dealing with optic neuropathy. In these cases, we suggest that COPD should be considered as an important risk factor for abnormal optic disc.

\section{References}

1. Halldin CN, Doney BC, Hnizdo E. Changes in prevalence of chronic obstructive pulmonary disease and asthma in the US population and associated risk factors. Chron Respir Dis 2015;12(1):47-60.

2. Regional COPD Working Group. COPD prevalence in 12 Asia-Pacific countries and regions: projections based on the COPD prevalence estimation model. Respirology 2003;8(2):192-198.

3. Agusti AG, Noguera A, Sauleda J, et al. Systemic effects of chronic obstructive pulmonary disease. Eur Respir J 2003;21(2):347-360.

4. Wouters EF, Creutzberg EC, Schols AM. Systemic effects in COPD. Chest 2002;121(5 Suppl):127S-130S.

5. Roland M, Bhowmik A, Sapsford RJ, et al. Sputum and plasma endothelin-1 levels in exacerbations of chronic obstructive pulmonary disease. Thorax 2006;56(1):30-35.

6. Ozer T, Altin R, Ugurbas $\mathrm{SH}$, et al. Color Doppler evaluation of the ocular arterial flow changes in chronic obstructive pulmonary disease. Eur J Radiol 2006;57(1):63-68.

7. Yanagi M, Kawasaki R, Wang JJ, et al. Vascular risk factors in glaucoma: a review. Clin Experiment Ophthalmol 2011;39(3):252-258.

8. Ozge C, Ozge A, Yilmaz A, Yalcinkaya DE, Calikoglu M. Cranial optic nerve involvement in patients with severe COPD. Respirology 2005;10(5):666-672.

9. Gupta PP, Sood S, Atreja A, Agarwal D. Assessment of visual evoked potentials in stable COPD patients with no visual impairment. Ann Thorac Med 2010;5(4):222-227.

10. Global Initiative for Chronic Obstructive Lung Disease (revised 2011). www.goldcopd.org/uploads/ users/files/GOLD_Report_2011_Feb21.pdf

11. Tsang CS, Chong SL, Ho CK, Li MF. Moderate to severe obstructive sleep apnea patients is associated with a higher incidence of visual field defect. Eye (Lond) 2006;20:38-42.

12. Kergoat H, Herard ME, Lemay M. RGC sensitivity to mild systemic hypoxia. Invest Ophthalmol Vis Sci 2006;47(12):5423-5427.

13. Flammer J. The vascular concept of glaucoma. Surv Ophthalmol 1994;38 suppl:S3-6.

14. Satilmis M, Orgul S, Doubler B, Flammer J. Rate of progression of glaucoma correlates with retrobulbar circulation and intraocular pressure. Am J Ophthalmol 2003;135(5):664-669.

15. Delaey C, Van De Voorde J. Regulatory mechanisms in the retinal and choroidal circulation. Ophthalmic Res 2000;32(6):249-256. 